\title{
Status of Understanding of the Disease and Self-Management Behavior among Patients with Hypertension
}

\author{
Ryoko Igarashi \\ Department of Medical Education, Tokyo Medical University, Tokyo, Japan \\ Email: igaryo@tokyo-med.ac.jp
}

How to cite this paper: Igarashi, R. (2019) Status of Understanding of the Disease and Self-Management Behavior among Patients with Hypertension. Health, 11, 905-923. https://doi.org/10.4236/health.2019.117073

Received: May 27, 2019

Accepted: July 15, 2019

Published: July 18, 2019

Copyright $\odot 2019$ by author(s) and Scientific Research Publishing Inc. This work is licensed under the Creative Commons Attribution International License (CC BY 4.0).

http://creativecommons.org/licenses/by/4.0/

\section{(c) (i) Open Access}

\begin{abstract}
Background: Hypertension is a lifestyle-related disease that has no subjective symptoms but could lead to severe complications. The goals of treatment for hypertension are to maintain blood pressure with medications and prevent complications. Measuring blood pressure at home regularly is critical for patients with hypertension. Purpose: This study aimed to 1) clarify the status of patients' understanding of hypertension and self-management, 2) identify nursing practices that could support patients with hypertension in making them aware of the need for understanding hypertension, monitoring blood pressure at home, and engaging in self-management. Method: A questionnaire survey on understanding of the disease and self-management behavior was conducted among patients with hypertension who regularly visited outpatient clinics. As for data analysis, descriptive statistics were computed for patients' attributes, clinical findings, understanding of the disease, and self-management behavior. Cross-tabulation and the chi-square test were used for each item on understanding of the disease and self-management behaviors. Results: Data were obtained from 150 participants $(93$ males and 57 females) with a mean age of 70 years. Patients with a higher understanding of the disease and those who were aware of the target blood pressure were more likely to engage in self-management behavior, including monitoring blood pressure at home, ensuring regular outpatient visits, and consulting with nurses or physicians. In addition, the percentage of patients who recognized the need to achieve their target blood pressure was higher among those who had a spouse and lived with their family. Discussion: Regular monitoring and recording of blood pressure at home are the most important self-management behaviors. Understanding of the disease and self-management behavior and support from the family are critical for maintaining appropriate self-management. Therefore, in cooperation with physi-
\end{abstract}


cians, nurses should provide health education to patients and their families to enable them to perform appropriate self-management. Health expenditures increase with age, especially in those aged 65 years and over. The prevalence of hypertension increases with age; patients aged 65 years and over need to engage in self-management to maintain their target blood pressure. Nurses should provide patients with hypertension aged 65 years and over with appropriate nursing care to enable them to measure and record their blood pressure at home appropriately and regularly. Appropriate nursing care may help reduce the health expenditure. This may help reduce the health expenditure.

\section{Keywords}

Self-Management Behavior, Hypertension Patient, Patient Education

\section{Introduction}

Hypertension is a lifestyle-related disease, and its prevalence increases with age. Hypertension is also known as a "silent killer" because severe complications can develop in the absence of subjective symptoms. High blood pressure increases the risk of onset of diseases such as coronary artery disease, cardiovascular system disorders, and cerebrovascular disorder, as well as the risk of mortality [1]. The goals of treatment for hypertension are basically to maintain optimal blood pressure with medications, achieve blood pressure control, and prevent complications. Therefore, patients are required to understand the need for measuring blood pressure, checking blood pressure daily, and monitoring their blood pressure to ensure that it is decreasing to the target they are attempting to reach.

The Japanese Society of Hypertension (JSH) published Guidelines for the Management of Hypertension 2014, which suggest the usefulness of monitoring blood pressure at home in improving the treatment continuation rate and evaluating if antihypertensive medications have been insufficiently or overly effective in reducing blood pressure as prescribed [1]. In addition to the blood pressure levels measured during medical examinations, the guidelines strongly recommend that blood pressure records from patients' home monitoring should be taken into consideration when deciding on a treatment plan. It strongly recommends that patients record their blood pressure regularly on record sheets.

Previous studies reported that blood pressure measurements from home are better predictors of the prognosis or onset of cardiovascular diseases as compared to the measurement of blood pressure during medical examinations [2]-[9]. Thus, it is critical for patients with hypertension to measure blood pressure at home and to continue to maintain records. Monitoring blood pressure at home by patients themselves may contribute to achieving targeted lower blood pressure levels; prevent the onset of diseases including coronary artery disease, cardiovascular disease, or cerebrovascular disease; maintain healthy life expec- 
tancy, and maintain/improve quality of life.

The present study aimed to 1) clarify the status of patients' understanding of hypertension and self-management, 2) identify nursing practices that could support patients with hypertension in making them aware of the need for understanding hypertension, monitoring blood pressure at home, and engaging in self-management.

\section{Methods}

1) Study period: April 1 to September 30, 2015.

2) Study site: The outpatient clinic of the Department of Cardiology at Tokyo Medical University Hospital.

3) Participants: Patients with hypertension who visited the outpatient cardiology clinic regularly Selection criteria of the participants were as follows: outpatients were diagnosed with hypertension, who regularly booked their next appointment and visited the hospital for follow-up examinations. Subjects were defined as patients with hypertension who regularly visited the outpatient cardiology clinic.

\section{4) Study context}

\section{a) Background of participants}

Sex, age, duration of disease, presence or absence of taking antihypertension medications, type of antihypertension medication, blood test results, height, weight, blood pressure during medical examination.

b) Survey on understanding of the disease and self-management behavior

A questionnaire developed by the authors was used to examine participants' understanding of the disease and self-management behavior. Items were scored on a dichotomous scale based on yes or no responses.

\section{5) Analysis methods}

Simple tabulation and descriptive analyses were used for examining patients' basic attributes and clinical findings. Additionally, descriptive statistics were computed for participants' understanding of the disease and self-management behavior. Cross-tabulation and the chi-square test were performed to examine each item pertaining to understanding of the disease and self-management behavior. Data were analyzed using IBM SPSS Statistics Ver.24.

\section{Ethical Considerations}

This study was approved by the Ethics Review Committee of the School of Nursing at Tokyo Medical University and Tokyo Medical University Hospital. The researcher orally explained the research objectives and participation details using instructions sheets to each participant. They were informed that participation in the study was voluntary and that they could withdraw consent at any time, even after agreeing to participate. They would receive no disadvantage as a result of leaving the study. It was also explained that personal information would be protected, that the research data would not be used for anything other than 
for the present study, and that all the data collected would be shredded after the completion of the study. Subsequently, those who agreed to participate in the study signed the consent form and completed the questionnaire. The survey was anonymous and participants did not need to fill in the questions they did not want to answer. The patients were asked to place the completed questionnaire in a collection box after completing their medical examination at the outpatient clinic.

\section{Results}

\subsection{Background of the Study Population}

Data were obtained from 150 participants (93 males and 57 females). The mean age was $70 \pm 10.6$ years, with a range of 46 - 92 years. The participants' educational level was distributed as follows: middle school: 16 (10.7\%), high school: 49 (32.7\%), college/vocational school: 26 (17.3\%), and undergraduate/graduate school: 58 (38.7\%). Further, 72 participants currently employed, and among them, 35 participants (38.7\%) were categorized as "office worker/public officials/staff member of association/teachers," $27(18 \%)$ as "runs private company/freelance business/small business," 1 participant $(0.7 \%)$ as "engaged in agricultural/fishing industry," and 9 participants (6\%) as "work part-time job." Additionally, 120 participants (80\%) were married, 30 (20\%) lived by themselves, and $120(80 \%)$ lived with their family.

The average disease duration was $8.4 \pm 5.8$ years. All participants took antihypertension medications and the average number of medications prescribed per participant was $2.1 \pm 1$. The average systolic and diastolic blood pressures were $139.1 \pm 18.1 \mathrm{mmHg}$ and $74.6 \pm 13 \mathrm{mmHg}$, respectively. The average body mass index (BMI) was $26.4 \pm 4 \mathrm{~kg} / \mathrm{m}^{2}$. Further, 91 (60.7\%) reported that they had a drinking habit, while 59 (39.3\%) reported that they did not have a drinking habit. Regarding smoking status, 20 (13.3\%) participants currently smoked, 63 (45.3\%) had smoked previously, and $62(41.4 \%)$ had never smoked. The average blood test results were as follows: total cholesterol level, $185.2 \pm 36.9$ $\mathrm{mg} / \mathrm{dl}$; triglycerides level, $144.6 \pm 74.9 \mathrm{mg} / \mathrm{dl}$; high-density lipoprotein (HDL) cholesterol level, $50.1 \pm 13.6 \mathrm{mg} / \mathrm{dl}$; low-density lipoprotein (LDL) cholesterol, $96.4 \pm 27 \mathrm{mg} / \mathrm{dl}$; urea nitrogen level, $16.1 \pm 4.6 \mathrm{mg} / \mathrm{dl}$; creatinine level, $1.5 \pm 8.4$ $\mathrm{mg} / \mathrm{dl}$; uric acid level, $5.7 \pm 1.3 \mathrm{mg} / \mathrm{dl}$; sodium level, $142 \pm 3.8 \mathrm{mEq} / \mathrm{L}$; potassium level, $4.4 \pm 3.5 \mathrm{mEq} / \mathrm{L}$; chlorine level, $105.9 \pm 2.4 \mathrm{mEq} / \mathrm{L}$; blood glucose level, $119.4 \pm 36 \mathrm{mg} / \mathrm{dl} ; \mathrm{HbA} 1 \mathrm{c}$ level, $6.2 \% \pm 0.9 \%$ (Table 1 ).

\subsection{Understanding of Hypertension}

For the question "Do you know what hypertension is?", 135 (90\%) participants answered "I do" and 15 (10\%) answered, "I don't." For the question "Do you know the risk factors that exacerbate hypertension?", 125 (83.4\%) answered "I do" and 25 (16.6\%) answered "I don't." For the question "Do you know the complications that can occur due to hypertension?", 118 (78.7\%) participants 
Table 1. Background of research.

\begin{tabular}{|c|c|}
\hline Age & $70.0 \pm 10.6$ \\
\hline \multicolumn{2}{|l|}{ Gender n (\%) } \\
\hline Male & $93(62 \%)$ \\
\hline Female & $57(38 \%)$ \\
\hline Disease duration & $8.4 \pm 5.8$ \\
\hline \multicolumn{2}{|l|}{ Taking antihypertensive medicine $\mathrm{n}(\%)$} \\
\hline Yes & $150(100 \%)$ \\
\hline No & $0(0 \%)$ \\
\hline Number of antihypertensive medicine & $2.1 \pm 1.0$ \\
\hline \multicolumn{2}{|l|}{ Educational Level n (\%) } \\
\hline Undergraduate/graduate school & $58(38.7 \%)$ \\
\hline College/vocational school & $26(17.3 \%)$ \\
\hline High school & $49(32.7 \%)$ \\
\hline Middle school & $16(10.7 \%)$ \\
\hline \multicolumn{2}{|l|}{ Employment n (\%) } \\
\hline Yes & $72(48 \%)$ \\
\hline No & $78(52 \%)$ \\
\hline \multicolumn{2}{|l|}{ Profession n (\%) } \\
\hline Office worker/public officials/staff member of association/teachers & $35(20 \%)$ \\
\hline Runs private company/freelance business/small business & $27(18 \%)$ \\
\hline Agricultural/fishing industry & $1(0.7 \%)$ \\
\hline \multicolumn{2}{|l|}{ Marriage n (\%) } \\
\hline Married & $120(80 \%)$ \\
\hline Unmarried/divorce & $30(20 \%)$ \\
\hline \multicolumn{2}{|l|}{ Living $n(\%)$} \\
\hline Living with family & $120(80 \%)$ \\
\hline Living alone & $30(20 \%)$ \\
\hline \multicolumn{2}{|l|}{ Drinking habit n (\%) } \\
\hline Yes & $91(60.7 \%)$ \\
\hline No & $59(39.3 \%)$ \\
\hline \multicolumn{2}{|l|}{ Smoking habit n (\%) } \\
\hline Yes (currently) & $20(13.3 \%)$ \\
\hline Yes (previously) & $68(45.3 \%)$ \\
\hline No & $62(41.4 \%)$ \\
\hline BMI (Body Mass Index) & $24.6 \pm 4.0$ \\
\hline Systolic Blood Pressure: SBP (mmHg) & $139.1 \pm 18.1$ \\
\hline Diastolic Blood Pressure: DBP (mmHg) & $74.6 \pm 13.0$ \\
\hline T-Cho (mg/dl) & $185.2 \pm 36.9$ \\
\hline $\mathrm{TG}(\mathrm{mg} / \mathrm{dl})$ & $144.6 \pm 74.9$ \\
\hline HDL-C (mg/dl) & $50.1 \pm 13.6$ \\
\hline LDL-C (mg/dl) & $96.4 \pm 27.0$ \\
\hline BUN (mg/dl) & $16.1 \pm 4.6$ \\
\hline Cre (mg/dl) & $1.5 \pm 8.4$ \\
\hline $\mathrm{UA}(\mathrm{mg} / \mathrm{dl})$ & $5.7 \pm 1.3$ \\
\hline $\mathrm{Na}(\mathrm{mEq} / \mathrm{L})$ & $142 \pm 3.8$ \\
\hline $\mathrm{K}(\mathrm{mEq} / \mathrm{L})$ & $4.4 \pm 3.5$ \\
\hline $\mathrm{Cl}(\mathrm{mEq} / \mathrm{L})$ & $105.9 \pm 2.4$ \\
\hline $\mathrm{BS}(\mathrm{mg} / \mathrm{dl})$ & $119.4 \pm 36.0$ \\
\hline HbAlc (\%) & $6.2 \pm 0.9$ \\
\hline
\end{tabular}


answered "I do" and 32 (21.3\%) answered "I don't." For the question "Do you know the symptoms of high blood pressure?", 104 (69.3\%) answered "I do" and $46(30.7 \%)$ answered "I don't." For the question "Do you know the need for reconsidering your lifestyle?", 127 (84.7\%) answered "I do" and 23 (15.3\%) answered "I don't." For the question "Do you know the types of prescribed drugs and their effects?", 120 (80\%) answered "I do" and 30 (20\%) answered "I don't." (Table 2).

\subsection{Self-Management Behaviors}

For the question "Do you have your own sphygmomanometer?", 142 (94.7\%) answered "I do" and 8 (5.3\%) answered "I don't." For the question "Do you measure your blood pressure at home?", 117 (78\%) answered "I do" and 33 (22\%) answered "I don't." For the question "Do you record your blood pressure in a notebook?", 91 (60.7\%) answered "I do" and 59 (39.3\%) answered "I don't." For the question "Do you know the appropriate blood pressure?", 140 (93.3\%) answered "I do" and 10 (6.7\%) answered "I don't." For the question "Do you visit your physicians as scheduled?", 149 (99.3\%) answered "I do" and 1 (0.7\%) answered "I don't." For the question "Do you show your blood pressure records to your physicians during the medical examination?", 67 (44.6\%) answered "I do" and 83 (55.4\%) answered "I don't." For the question "Do you consult with physicians or nurses?", 131 (87.3\%) answered "I do" and 19 (12.7\%) answered "I don't." ('Table 3).

\subsection{Cross-Tabulation}

1) Understanding of each item on knowledge of disease/understanding of disease and self-management behaviors

Table 2. Understanding of hypertension $(n=150)$.

\begin{tabular}{|c|c|c|c|}
\hline & & Frequency & $(\%)$ \\
\hline \multirow{2}{*}{ Do you know what hypertension is? } & I do & 135 & $90.0 \%$ \\
\hline & I don't & 15 & $10.0 \%$ \\
\hline \multirow{2}{*}{ Do you know the risk factors that exacerbate hypertension? } & I do & 125 & $83.4 \%$ \\
\hline & I don't & 25 & $16.6 \%$ \\
\hline \multirow{2}{*}{$\begin{array}{l}\text { Do you know the complications that can occur due to } \\
\text { hypertension? }\end{array}$} & I do & 118 & $78.7 \%$ \\
\hline & I don't & 32 & $21.3 \%$ \\
\hline \multirow[b]{2}{*}{ Do you know the symptoms of high blood pressure? } & I do & 104 & $69.3 \%$ \\
\hline & I don't & 46 & $30.7 \%$ \\
\hline \multirow{2}{*}{ Do you know the need for reconsidering your lifestyle? } & I do & 127 & $84.7 \%$ \\
\hline & I don't & 23 & $15.3 \%$ \\
\hline \multirow{2}{*}{ Do you know the types of prescribed drugs and their effects? } & I do & 120 & $80.0 \%$ \\
\hline & I don't & 30 & $20.0 \%$ \\
\hline
\end{tabular}


Table 3. Self-management behaviors $(n=150)$.

\begin{tabular}{lccc}
\hline & & Frequency & $(\%)$ \\
\hline Do you have your own sphygmomanometer? & I do & 142 & $94.7 \%$ \\
Do you measure your blood pressure at home? & I don't & 8 & $5.3 \%$ \\
& I do & 117 & $78.0 \%$ \\
Do you record your blood pressure in a notebook? & I don't & 33 & $22.0 \%$ \\
& I do & 91 & $60.7 \%$ \\
Do you know the appropriate blood pressure? & I don't & 59 & $39.3 \%$ \\
& I do & 140 & $93.3 \%$ \\
Do you visit your physician as scheduled? & I don't & 10 & $6.7 \%$ \\
Do you show your blood pressure records to your & I do & 149 & $99.3 \%$ \\
physician during the medical examination? & I don't & 1 & $0.7 \%$ \\
Do you consult with physicians or nurses? & I do & 67 & $44.6 \%$ \\
& I don't & 83 & $55.4 \%$ \\
\hline
\end{tabular}

Understanding of the disease ( 7 items) and self-management behaviors (5 items) were cross-tabulated (Table 4). "Understanding of hypertension" was significantly associated with "recording blood pressure," "showing the records to physicians during medical examination," and "consulting with physicians or nurses." "Understanding of the risk factors that exacerbate hypertension" was significantly associated with "measuring blood pressure at home," "recording blood pressure," and "consulting with physicians or nurses." "Understanding of complications led to by hypertension" was significantly associated with "measuring blood pressure at home," "recording blood pressure," and "consulting with physicians or nurses." "Understanding of subjective symptoms when experiencing high blood pressure" was significantly associated with "measuring blood pressure at home" and "consulting with physicians or nurses." "The necessity of reconsidering lifestyle" was significantly associated with "measuring blood pressure at home," "showing the records to physicians during medical examination," and "consulting with physicians or nurses." "Understanding of prescribed medications" was significantly associated with "measuring blood pressure at home" and "consulting with physicians or nurses." "Understanding of target blood pressure" was significantly associated with "measuring blood pressure at home."

\section{a) Understanding of hypertension and self-management behavior}

When examining the association between "understanding of hypertension" and "recording blood pressure," participants who reported "yes" to the former had a significantly higher percentage of recording their blood pressure (94.5\%, adjusted residual $=2.2$ ). Pertaining to "showing the records to physicians at a medical examination," participants who reported that they had an understanding of hypertension had a significantly higher percentage of showing the records 
Table 4. Relationship between knowledge and understanding of disease and self-management behavior.

\begin{tabular}{|c|c|c|c|c|}
\hline \multirow[b]{2}{*}{$\begin{array}{c}\text { Understanding of } \\
\text { knowledge/understanding } \\
\text { of disease }\end{array}$} & \multicolumn{4}{|c|}{ Self-management behavior } \\
\hline & $\begin{array}{l}\text { Measuring blood } \\
\text { pressure at home }\end{array}$ & $\begin{array}{l}\text { Recording blood } \\
\text { pressure }\end{array}$ & $\begin{array}{l}\text { Showing the records } \\
\text { to physicians } \\
\text { during medical } \\
\text { examination }\end{array}$ & $\begin{array}{l}\text { Consulting with } \\
\text { physicians } \\
\text { or nurses }\end{array}$ \\
\hline Understanding of hypertension & & $\begin{array}{c}\text { Asymptotic significance } \\
\text { probability }(0.027) \\
\mathrm{X}^{2}(2)=4.882\end{array}$ & $\begin{array}{c}\text { Asymptotic significance } \\
\text { probability }(0.026) \\
\mathrm{X}^{2}(2)=4.973\end{array}$ & $\begin{array}{c}\text { Asymptotic significance } \\
\text { probability }(0.000) \\
\mathrm{X}^{2}(2)=18.785\end{array}$ \\
\hline $\begin{array}{l}\text { Understanding of the risk factors } \\
\text { that exacerbate hypertension }\end{array}$ & $\begin{array}{c}\text { Asymptotic significance } \\
\text { probability }(0.001) \\
\mathrm{X}^{2}(2)=11.818\end{array}$ & $\begin{array}{c}\text { Asymptotic significance } \\
\text { probability }(0.028) \\
\mathrm{X}^{2}(2)=4.846\end{array}$ & $\begin{array}{c}\text { Asymptotic significance } \\
\text { probability }(0.034) \\
\mathrm{X}^{2}(2)=4.480\end{array}$ & $\begin{array}{c}\text { Asymptotic significance } \\
\text { probability }(0.000) \\
\mathrm{X}^{2}(2)=11.222\end{array}$ \\
\hline $\begin{array}{l}\text { Understanding of complications } \\
\text { led to by hypertension }\end{array}$ & $\begin{array}{c}\text { Asymptotic significance } \\
\text { probability }(0.022) \\
\mathrm{X}^{2}(2)=5.243\end{array}$ & & $\begin{array}{c}\text { Asymptotic significance } \\
\text { probability }(0.022) \\
\mathrm{X}^{2}(2)=5.243\end{array}$ & $\begin{array}{c}\text { Asymptotic significance } \\
\text { probability }(0.000) \\
\mathrm{X}^{2}(2)=14.099\end{array}$ \\
\hline $\begin{array}{l}\text { Understanding of subjective } \\
\text { symptoms when experiencing } \\
\text { high blood pressure }\end{array}$ & $\begin{array}{c}\text { Asymptotic significance } \\
\text { probability }(0.000) \\
\mathrm{X}^{2}(2)=14.408\end{array}$ & & & $\begin{array}{c}\text { Asymptotic significance } \\
\text { probability }(0.002) \\
\mathrm{X}^{2}(2)=9.279\end{array}$ \\
\hline $\begin{array}{l}\text { The necessity of reconsidering } \\
\text { lifestyle }\end{array}$ & $\begin{array}{c}\text { Asymptotic significance } \\
\text { probability }(0.007) \\
\mathrm{X}^{2}(2)=7.303\end{array}$ & & $\begin{array}{c}\text { Asymptotic significance } \\
\text { probability }(0.042) \\
\mathrm{X}^{2}(2)=4.141\end{array}$ & $\begin{array}{c}\text { Asymptotic significance } \\
\text { probability }(0.000) \\
\mathrm{X}^{2}(2)=18.737\end{array}$ \\
\hline $\begin{array}{l}\text { Understanding of prescribed } \\
\text { medications }\end{array}$ & $\begin{array}{c}\text { Asymptotic significance } \\
\text { probability }(0.000) \\
\mathrm{X}^{2}(2)=13.296\end{array}$ & & & $\begin{array}{c}\text { Asymptotic significance } \\
\text { probability }(0.034) \\
\mathrm{X}^{2}(2)=4.478\end{array}$ \\
\hline $\begin{array}{l}\text { Understanding of target } \\
\text { blood pressure }\end{array}$ & $\begin{array}{c}\text { Asymptotic significance } \\
\text { probability }(0.003) \\
\mathrm{X}^{2}(2)=9.016\end{array}$ & & & \\
\hline $\begin{array}{l}\mathrm{p}<0.05, \text { versus each respective } \\
\text { countenrparts }\end{array}$ & & & & \\
\hline
\end{tabular}

$(95.5 \%$, adjusted residual $=2.2)$. Pertaining to "consulting with physicians or nurses," those who that they had an understanding of hypertension had a significantly higher percentage of consulting with physicians or nurses (93.9\%, adjusted residual $=4.3, \mathrm{P}=0.000)$ (Table 5).

b) Understanding the risk factors that exacerbate hypertension and self-management behavior

Among those who understood the risk factors that exacerbate hypertension, a significantly higher percentage reported measuring their blood pressure at home $(88.9 \%$, adjusted residual $=3.4)$, recording their blood pressure $(92.3 \%$, adjusted residual $=2.2$ ), showing the records to the physicians during a medical examination $(89.6 \%$, adjusted residual $=2.1)$, and consulting with physicians or nurses $(87 \%$, adjusted residual $=3.3)$ (Table 6$)$.

c) Understanding of complications caused by hypertension and self-management behavior

Among those who understood the complications caused by hypertension, a significantly higher percentage reported measuring blood pressure at home 
Table 5. Understanding of hypertension and self-management behavior.

\begin{tabular}{|c|c|c|c|c|}
\hline \multicolumn{4}{|c|}{ Recording blood pressure } & \multirow{2}{*}{$\mathrm{X}^{2}$ Test } \\
\hline \multicolumn{2}{|c|}{ Understanding of hypertension } & Yes & No & \\
\hline Yes & frequency (\%) & $86(94.5 \%)$ & $21(80.8 \%)$ & \multirow{4}{*}{$\mathrm{P}=0.027$} \\
\hline \multirow{4}{*}{ No } & adjusted residual & 2.2 & -2.2 & \\
\hline & frequency $(\%)$ & $5(5.5 \%)$ & $5(19.2 \%)$ & \\
\hline & adjusted residual & -2.2 & 2.2 & \\
\hline & & \multicolumn{2}{|c|}{ medical examination } & \multirow{2}{*}{$\mathrm{X}^{2}$ Test } \\
\hline \multicolumn{2}{|c|}{ Understanding of hypertension } & Yes & No & \\
\hline Yes & frequency (\%) & $64(95.5 \%)$ & $63(84 \%)$ & \multirow{4}{*}{$\mathrm{P}=0.026$} \\
\hline \multirow{4}{*}{ No } & adjusted residual & 2.2 & -2.2 & \\
\hline & frequency (\%) & $3(4.5 \%)$ & $12(16 \%)$ & \\
\hline & \multirow[t]{2}{*}{ adjusted residual } & -2.2 & 2.2 & \\
\hline & & \multicolumn{2}{|c|}{ Consulting with physicians or nurses } & \multirow{2}{*}{$\mathrm{X}^{2}$ Test } \\
\hline \multicolumn{2}{|c|}{ Understanding of hypertension } & Yes & No & \\
\hline \multirow[t]{2}{*}{ Yes } & frequency (\%) & $123(93.9 \%)$ & $11(61.1 \%)$ & \multirow{4}{*}{$\mathrm{P}=0.000$} \\
\hline & adjusted residual & 4.3 & -4.3 & \\
\hline \multirow[t]{2}{*}{ No } & frequency (\%) & $8(6.1 \%)$ & $7(38.9 \%)$ & \\
\hline & adjusted residual & -4.3 & 4.3 & \\
\hline
\end{tabular}

Table 6. Understanding the risk factors that exacerbate hypertension and self-management behavior.

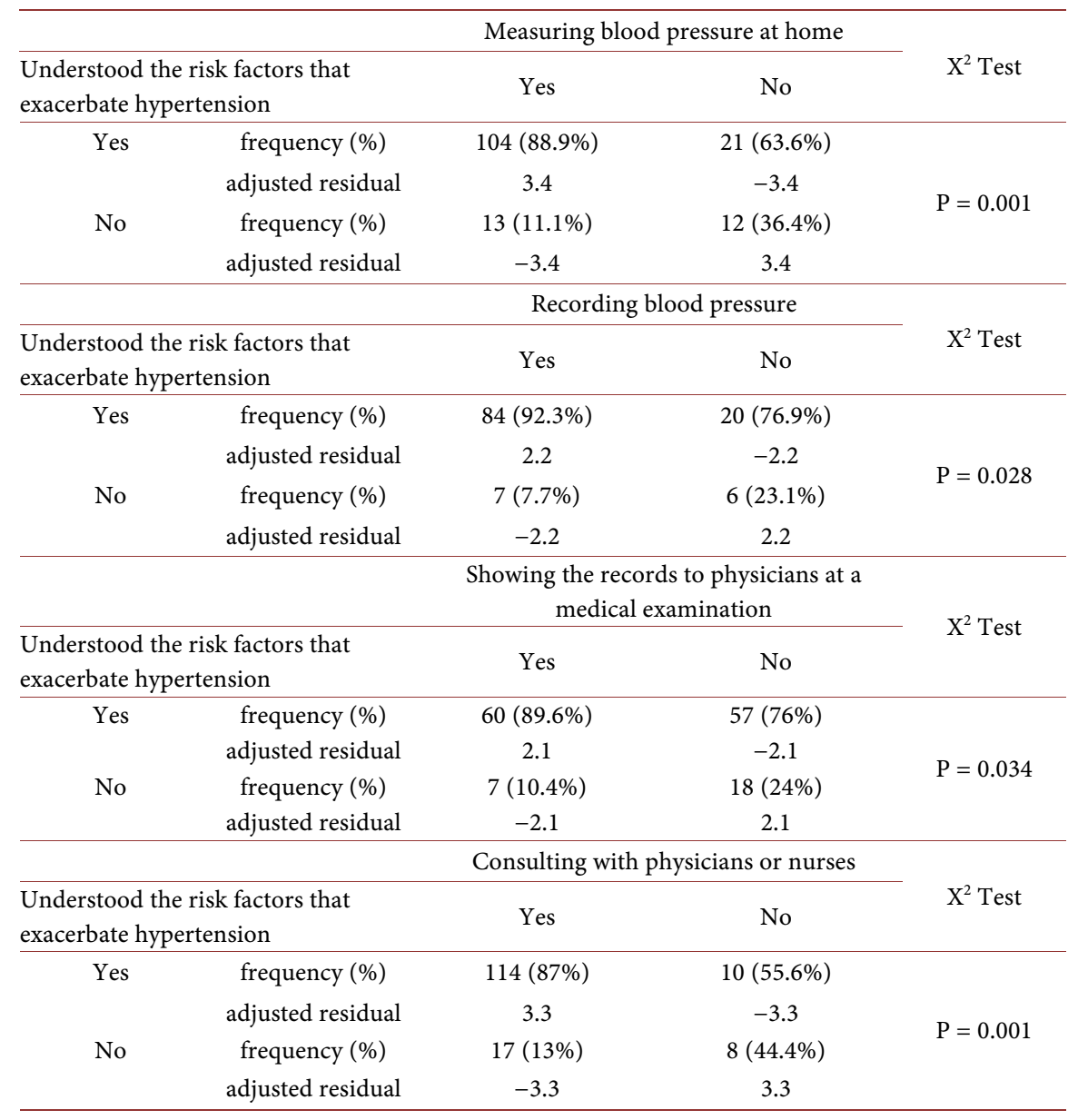


$(82.9 \%$, adjusted residual $=2.4)$, showing the records to their physicians during medical examinations $(88.6 \%$, adjusted residual $=2.3)$, and consulting with physicians or nurses $(83.2 \%$, adjusted residual $=3.8)($ Table 7$)$.

d) Understanding of subjective symptoms of high blood pressure and self-management behavior

Among those who understood symptoms of high blood pressure, a significantly higher percentage reported measuring blood pressure at home $(76.9 \%$, adjusted residual $=3.8)$ and consulting with physicians or nurses $(74 \%$, adjusted residual $=3.0)($ Table 8$)$.

e) Understanding of the need for reconsidering lifestyle and self management behavior

Among those who understood the need for reconsidering their lifestyle, a significantly higher percentage reported measuring blood pressure at home $(88.9 \%$, adjusted residual $=2.7$ ), showing their records to their physicians during medical examinations $(91 \%$, adjusted residual $=2.0)$, and consulting with physicians or nurses $(89.3 \%$, adjusted residual $=4.3)$ (Table 9).

f) Understanding of prescribed medications and self-management behavior

Among those who understood prescribed medications, a significantly higher percentage reported measuring blood pressure at home $(86.3 \%$, adjusted residual $=3.6)$ and consulting with physicians or nurses $(82.4 \%$, adjusted residual $=$

Table 7. Understanding of complications caused by hypertension and self-management behavior.

\begin{tabular}{|c|c|c|c|c|}
\hline & & \multicolumn{2}{|c|}{ Measuring blood pressure at home } & \multirow[b]{2}{*}{$\mathrm{X}^{2}$ Test } \\
\hline \multicolumn{2}{|c|}{$\begin{array}{l}\text { Understood the complications caused } \\
\text { by hypertension }\end{array}$} & Yes & No & \\
\hline Yes & Frequency (\%) & $97(82.9 \%)$ & $21(63.6 \%)$ & \multirow{3}{*}{$\mathrm{P}=0.022$} \\
\hline \multirow[t]{3}{*}{ No } & $\begin{array}{c}\text { Adjusted residual } \\
\text { Frequency (\%) }\end{array}$ & $\begin{array}{c}2.4 \\
20(17.1 \%)\end{array}$ & $\begin{array}{c}-2.4 \\
12(36.4 \%)\end{array}$ & \\
\hline & Adjusted residual & -2.4 & 2.4 & \\
\hline & & $\begin{array}{r}\text { Showing the } \mathrm{r} \\
\text { medi }\end{array}$ & $\begin{array}{l}\text { physicians at a } \\
\text { nation }\end{array}$ & \multirow{2}{*}{$\mathrm{X}^{2}$ Test } \\
\hline $\begin{array}{l}\text { Understood } \\
\text { by hyperten }\end{array}$ & mplications caused & Yes & No & \\
\hline Yes & Frequency (\%) & $58(86.6 \%)$ & $53(70.7 \%)$ & \multirow{4}{*}{$\mathrm{P}=0.022$} \\
\hline \multirow{4}{*}{ No } & Adjusted residual & 2.3 & -2.3 & \\
\hline & Frequency (\%) & $9(13.4 \%)$ & $22(29.3 \%)$ & \\
\hline & Adjusted residual & -2.3 & 2.3 & \\
\hline & & \multicolumn{2}{|c|}{ Consulting with physicians or nurses } & \multirow[b]{2}{*}{$\mathrm{X}^{2}$ Test } \\
\hline $\begin{array}{l}\text { Understood } \\
\text { by hyperten }\end{array}$ & mplications caused & Yes & No & \\
\hline \multirow[t]{2}{*}{ Yes } & Frequency (\%) & $109(83.2 \%)$ & $8(44.4 \%)$ & \multirow{4}{*}{$\mathrm{P}=0.000$} \\
\hline & Adjusted residual & 3.8 & -3.8 & \\
\hline \multirow[t]{2}{*}{ No } & Frequency (\%) & $22(16.8 \%)$ & $10(55.6 \%)$ & \\
\hline & Adjusted residual & -3.8 & 3.8 & \\
\hline
\end{tabular}


Table 8. Understanding of subjective symptoms of high blood pressure and self-management behavior.

\begin{tabular}{|c|c|c|c|c|}
\hline & & \multicolumn{2}{|c|}{ Measuring blood pressure at home } & \multirow[b]{2}{*}{$\mathrm{X}^{2}$ Test } \\
\hline \multicolumn{2}{|c|}{$\begin{array}{l}\text { Understanding of subjective } \\
\text { symptoms of high blood pressure }\end{array}$} & Yes & No & \\
\hline Yes & Frequency (\%) & $90(76.9 \%)$ & $14(42.4 \%)$ & \multirow{4}{*}{$\mathrm{P}=0.000$} \\
\hline \multirow{4}{*}{ No } & Adjusted residual & 3.8 & -3.8 & \\
\hline & Frequency (\%) & $27(23.1 \%)$ & $19(57.6 \%)$ & \\
\hline & Adjusted residual & -3.8 & 3.8 & \\
\hline & & Consulting $\mathrm{V}$ & ians or nurses & \multirow[b]{2}{*}{$\mathrm{X}^{2}$ Test } \\
\hline $\begin{array}{l}\text { Understand } \\
\text { symptoms o }\end{array}$ & $\begin{array}{l}\text { subjective } \\
\text { blood pressure }\end{array}$ & Yes & No & \\
\hline \multirow[t]{2}{*}{ Yes } & Frequency $(\%)$ & $97(74 \%)$ & $7(38.9 \%)$ & \multirow{4}{*}{$\mathrm{P}=0.002$} \\
\hline & Adjusted residual & 3 & -3 & \\
\hline \multirow[t]{2}{*}{ No } & Frequency (\%) & $34(26 \%)$ & $11(61.1 \%)$ & \\
\hline & Adjusted residual & -3 & 3 & \\
\hline
\end{tabular}

Table 9. Understanding of the need for reconsidering lifestyle and self-management behavior.

\begin{tabular}{|c|c|c|c|c|}
\hline & & \multicolumn{2}{|c|}{ Measuring blood pressure at home } & \multirow[b]{2}{*}{$\mathrm{X}^{2}$ Test } \\
\hline \multicolumn{2}{|c|}{$\begin{array}{l}\text { Understanding of the need for } \\
\text { reconsidering lifestyle }\end{array}$} & Yes & No & \\
\hline Yes & Frequency (\%) & $104(88.9 \%)$ & $23(69.7 \%)$ & \multirow{3}{*}{$\mathrm{P}=0.007$} \\
\hline \multirow[t]{3}{*}{ No } & $\begin{array}{l}\text { Adjusted residual } \\
\text { Frequency (\%) }\end{array}$ & $\begin{array}{c}2.7 \\
13(11.1 \%)\end{array}$ & $\begin{array}{c}-2.7 \\
10(30.3 \%)\end{array}$ & \\
\hline & Adjusted residual & -2.7 & 2.7 & \\
\hline & & \multicolumn{2}{|c|}{$\begin{array}{l}\text { Showing the records to physicians at a } \\
\text { medical examination }\end{array}$} & \multirow{2}{*}{$\mathrm{X}^{2}$ Test } \\
\hline \multicolumn{2}{|c|}{$\begin{array}{l}\text { Understanding of the need for } \\
\text { reconsidering lifestyle }\end{array}$} & Yes & No & \\
\hline Yes & Frequency (\%) & $61(91 \%)$ & $59(78.7 \%)$ & \multirow{4}{*}{$\mathrm{P}=0.042$} \\
\hline \multirow{4}{*}{ No } & Adjusted residual & 2 & -2 & \\
\hline & Frequency (\%) & $6(9 \%)$ & $16(21.3 \%)$ & \\
\hline & Adjusted residual & -2 & 2 & \\
\hline & & Consulting w & ians or nurses & \multirow[b]{2}{*}{$\mathrm{X}^{2}$ Test } \\
\hline $\begin{array}{l}\text { Understand } \\
\text { reconsiderir }\end{array}$ & $\begin{array}{l}\text { the need for } \\
\text { tyle }\end{array}$ & Yes & No & \\
\hline \multirow[t]{2}{*}{ Yes } & Frequency (\%) & $117(89.3 \%)$ & $9(50 \%)$ & \multirow{4}{*}{$\mathrm{P}=0.000$} \\
\hline & Adjusted residual & 4.3 & -4.3 & \\
\hline \multirow[t]{2}{*}{ No } & Frequency (\%) & $14(10.7 \%)$ & $9(50 \%)$ & \\
\hline & Adjusted residual & -4.3 & 4.3 & \\
\hline
\end{tabular}

\section{1). (Table 10).}

g) Understanding of target blood pressure and self-management behavior Among those who understood target blood pressure, a significantly higher percentage reported measuring blood pressure at home (96.6\%, adjusted residual = 3.0) (Table 11). 
Table 10. Understanding of prescribed medications and self-management behavior.

\begin{tabular}{|c|c|c|c|c|}
\hline & & \multicolumn{2}{|c|}{ Measuring blood pressure at home } & \multirow[b]{2}{*}{$\mathrm{X}^{2}$ Test } \\
\hline \multicolumn{2}{|c|}{$\begin{array}{l}\text { Understanding of prescribed } \\
\text { medications }\end{array}$} & Yes & No & \\
\hline Yes & Frequency $(\%)$ & $101(86.3 \%)$ & $19(57.6 \%)$ & \multirow{4}{*}{$\mathrm{P}=0.000$} \\
\hline \multirow{4}{*}{ No } & Adjusted residual & 3.6 & -3.6 & \\
\hline & Frequency (\%) & $16(13.7 \%)$ & $14(42.4 \%)$ & \\
\hline & Adjusted residual & -3.6 & 3.6 & \\
\hline & & Consulting $\mathrm{w}$ & ians or nurses & \multirow[b]{2}{*}{$\mathrm{X}^{2}$ Test } \\
\hline $\begin{array}{l}\text { Understand } \\
\text { medication }\end{array}$ & prescribed & Yes & No & \\
\hline Yes & Frequency (\%) & $108(82.4 \%)$ & $11(61.1 \%)$ & \multirow{4}{*}{$\mathrm{P}=0.034$} \\
\hline \multirow{3}{*}{ No } & Adjusted residual & 2.1 & -2.1 & \\
\hline & Frequency (\%) & $23(17.6 \%)$ & $7(38.9 \%)$ & \\
\hline & Adjusted residual & -2.1 & 2.1 & \\
\hline
\end{tabular}

Table 11. Understanding of target blood pressure and self-management behavior.

\begin{tabular}{|c|c|c|c|c|}
\hline \multirow{2}{*}{\multicolumn{2}{|c|}{$\begin{array}{l}\text { Understanding of target blood } \\
\text { pressure }\end{array}$}} & \multicolumn{2}{|c|}{ Measuring blood pressure at home } & \multirow[b]{2}{*}{$\mathrm{X}^{2}$ Test } \\
\hline & & Yes & No & \\
\hline Yes & Frequency (\%) & $113(96.6 \%)$ & $27(81.8 \%)$ & \multirow{3}{*}{$\mathrm{P}=0.003$} \\
\hline \multirow[t]{2}{*}{ No } & $\begin{array}{l}\text { Adjusted residual } \\
\text { Frequency (\%) }\end{array}$ & $\begin{array}{c}3 \\
4(3.4 \%)\end{array}$ & $\begin{array}{c}-3 \\
6(18.2 \%)\end{array}$ & \\
\hline & Adjusted residual & -3 & 3 & \\
\hline
\end{tabular}

2) Basic attributes of participants and their understanding of the disease, and self-management behavior

Cross-tabulation was performed between the basic attributes of participants and their understanding of the disease ( 7 items), and their self-management behaviors (5 items) (Table 12). There was a significant association between educational level and three items pertaining to understanding of the disease (understanding of subjective symptoms of high blood pressure, understanding of the need for reconsidering their lifestyle, and understanding of prescribed medications), and one item on self-management behaviors (measuring blood pressure at home). Living situation was significantly associated with the understanding of target blood pressure. Occupational status was significantly associated with measuring blood pressure at home. Age was significantly associated with the understanding of prescribed medications.

\section{a) Basic attributes of participants and understanding of the disease}

Participants with an educational attainment level of up to middle school had a significantly higher percentage of those reporting that they did not have an understanding of the symptoms of high blood pressure $(62.5 \%$, adjusted residual $=$ 2.9 ), need for reconsidering their lifestyle $(43.8 \%$, adjusted residual $=3.3)$, and prescribed medications $(50 \%$, adjusted residual $=3.2$ ). Housewives $/$ husbands had a significantly higher percentage of individuals who measured blood pressure at home $(96.6 \%$, adjusted residual $=2.7)$. Participants aged 65 years and 
Table 12. Basic attributes of participants and their understanding of the disease, and self-management behavior.

\begin{tabular}{|c|c|c|c|c|}
\hline \multirow[b]{2}{*}{$\begin{array}{l}\text { Self-management } \\
\text { behavior } \\
\text { Understanding of } \\
\text { knowledge/disease }\end{array}$} & \multicolumn{4}{|c|}{ Participant background } \\
\hline & $\begin{array}{l}\text { Educational } \\
\text { Level }\end{array}$ & $\begin{array}{c}\text { Living } \\
\text { (living } \\
\text { alone/living } \\
\text { with family) }\end{array}$ & Age & Profession \\
\hline $\begin{array}{l}\text { Understanding of } \\
\text { subjective symptoms } \\
\text { when experiencing } \\
\text { high blood pressure }\end{array}$ & $\begin{array}{c}\text { Asymptotic } \\
\text { significance } \\
\text { probability }(0.013) \\
\mathrm{X}^{2}(2)=10.7888\end{array}$ & & & \\
\hline $\begin{array}{l}\text { The necessity of } \\
\text { reconsidering lifestyle }\end{array}$ & $\begin{array}{c}\text { Asymptotic } \\
\text { significance } \\
\text { probability }(0.007) \\
\mathrm{X}^{2}(2)=12.202\end{array}$ & & & \\
\hline $\begin{array}{l}\text { Understanding of } \\
\text { prescribed medications }\end{array}$ & $\begin{array}{c}\text { Asymptotic } \\
\text { significance } \\
\text { probability }(0.017) \\
\mathrm{X}^{2}(2)=10.232\end{array}$ & & $\begin{array}{c}\text { Asymptotic } \\
\text { significance } \\
\text { probability } \\
(0.049) \\
\mathrm{X}^{2}(2)=6.034\end{array}$ & \\
\hline $\begin{array}{l}\text { Understanding of target } \\
\text { blood pressure }\end{array}$ & & $\begin{array}{c}\text { Asymptotic } \\
\text { significance } \\
\text { probability } \\
(0.014) \\
\mathrm{X}^{2}(2)=6.027\end{array}$ & & \\
\hline $\begin{array}{l}\text { Measuring blood } \\
\text { pressure at home }\end{array}$ & $\begin{array}{c}\text { Asymptotic } \\
\text { significance } \\
\text { probability }(0.021) \\
\mathrm{X}^{2}(2)=9.778\end{array}$ & & & $\begin{array}{c}\text { Asymptotic } \\
\text { significance } \\
\text { probability } \\
(0.014) \\
\mathrm{X}^{2}(2)=15.996\end{array}$ \\
\hline
\end{tabular}

younger had a significantly higher percentage of individuals who reported understanding prescribed medications $(91.1 \%$, adjusted residual $=2.2)$ (Table 13).

b) Basic attributes of participants and self-management behaviors

Participants who lived with their family reported a significantly higher percentage of understanding target blood pressure $(95.8 \%$, adjusted residual $=2.5)$ (Table 14).

\section{Discussion}

When considering the association between self-management behaviors and understanding of the disease, participants who measured their blood pressure at home were more likely to understand hypertension, the risk factors that exacerbate it, related complications, subjective symptoms, types and effects of prescribed medications, and the importance of lowering blood pressure to targeted values. According to the recommendations of the Guidelines for the Management of Hypertension 2014, better understanding and self-management has been achieved when physicians stress on the importance of measuring blood pressure and providing guidance at each visit for patients to measure at home 
Table 13. Basic attributes of participants and understanding of the disease.

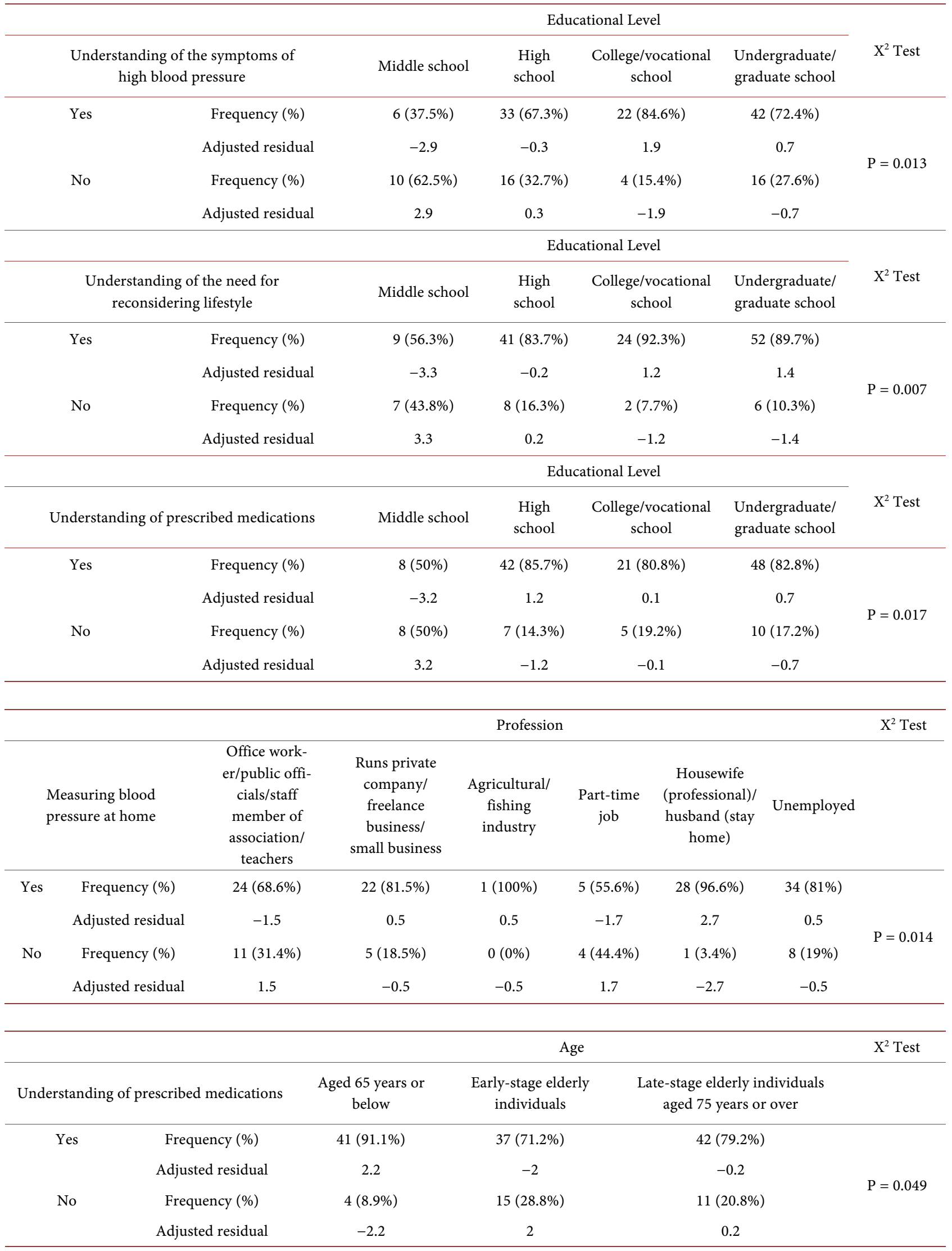


Table 14. Basic attributes of participants and self-management behavior.

\begin{tabular}{ccccc}
\hline & & \multicolumn{2}{c}{ Living } & \multirow{2}{*}{$\mathrm{X}^{2}$ Test } \\
\cline { 1 - 3 } \multicolumn{2}{c}{ Understanding target blood pressure } & Living alone & Living with family & \\
\cline { 1 - 3 } Yes & $\begin{array}{c}\text { Frequency (\%) } \\
\text { adjusted residual }\end{array}$ & $25(83.3 \%)$ & $115(95.8 \%)$ & \\
& Frequency (\%) & $5(16.7 \%)$ & $5(4.2 \%)$ & \multirow{2}{*}{$\mathrm{P}=0.014$} \\
No & $\begin{array}{c}\text { adjusted residual } \\
\end{array}$ & 2.5 & -2.5 & \\
\hline
\end{tabular}

[1]. In addition, due to the long duration of hypertension ( $8.4 \pm 5.8$ years) in the present sample, most participants understood the disease, measured their blood pressure at home, and understood the need to achieve the target blood pressure. In addition, the present participants' mean age was 70 years, which suggests that the age at which physiological functions decrease and the risk of exacerbating hypertension or developing complications increases may also contribute to patients' tendency to measure blood pressure at home. On the other hand, in the present sample, participants who recorded their blood pressure at home comprised only those who responded that they understood the risk factors that exacerbate hypertension. Imai et al. suggested that home records could help medical staff to understand their blood pressure [10]. Okubo et al. also reported that blood pressure measurements from home were a better predictor to assess prognosis, as compared to blood pressure measurements taken during medical examinations [11]. Thus, physicians need to provide patients with clear explanations about measuring and recording blood pressure at home and they should supply a record notebook during medical examinations. After seeing the physician, nurses need to provide patients with health guidance on how to measure and record blood pressure at home corresponding to their individual abilities, such as the level of patients' understanding or their actual practice of self-management behaviors. In other words, it is critical for physicians and nurses to cooperate and strengthen health guidance depending on patients' individual characteristics in order to enable them to measure and record blood pressure appropriately at home on a daily basis.

In this study, many participants reported that they consulted with physicians or nurses. This may indicate that they recognized the need for understanding their disease and their physical conditions, and they were aware that it takes time to recover once the disease aggravates and that age-related changes affect their health. This result also suggests that providing health guidance thoroughly with the cooperation of physicians and nurses would further facilitate patients' consultation with health personnel and their ability to engage in appropriate self-management behaviors. During medical examinations, physicians and nurses can evaluate the self-management situation at home through the record notebooks or consultation. They could use this information to provide words of encouragement and appreciation of patients' efforts, and to provide positive feedback on what patients have done, which would in turn increase patients' 
confidence and motivate their self-management. Thus, healthcare professionals can foster patients' continuation of self-management and increase their self-efficacy.

Considering the association between the participants' basic attributes and understanding of the disease, and their self-management behaviors, the present study found that participants with higher education tended to understand the disease and measure blood pressure at home. Such patients may be able to obtain information about the disease and methods of self-management more effectively, which could deepen their knowledge about the disease and self-management. Based on this knowledge, they may have a greater ability to understand and identify important self-management that match their lifestyle.

Kubota et al. analyzed lifetime risk of developing cardiovascular diseases according to educational achievement, using results of a large-scale cohort study that followed 13,948 participants for 26 years [12]. They reported that participants with higher educational attainment had a lower risk of developing cardiovascular diseases, and those with an educational attainment of graduate school level showed the lowest risk. These findings suggest that educational level may possibly be a factor related to health disparities, and they support our findings that participants with higher educational level understood the disease and measured blood pressure at home.

Participants who lived with their family were more likely to understand the need for achieving their target blood pressure. Previous studies indicated that existence and support from the family are necessary for patients to perform appropriate self-management [13] [14] [15] [16] [17]. Hara et al. claimed that living with their spouse and having family bonds offered a physiologically stable treatment environment for patients and helped maintain a high self-esteem level, leading to better self-management [18]. In the present study, 120 participants (80\%) who had a spouse and live with their family were in an environment where they received support from their family for self-management. This may explain the higher percentage of understanding the need for achieving their target blood pressure among this group of participants. In addition, target blood pressure is an explicitly "visualized" value that families can easily understand, and they may be able to give specific advice or help depending on patients' situation. Therefore, patients living with their family had higher percentage in understanding the need for achieving their target blood pressure.

In Japan, the health expenditures on hypertension were 1,798,100,000,000 JPY in 2016. Further, health expenditures increase with age, especially in those aged 65 years and over [19]. The prevalence of hypertension increases with age; thus, especially patients aged 65 years and over need to engage in self-management to maintain their target blood pressure. Accordingly, nurses should provide patients with hypertension aged 65 years and over with appropriate nursing care to enable them to measure and record their blood pressure at home appropriately and regularly. This practice may in turn help reduce the health expenditure in this group. 
The Guidelines for the Management of Hypertension 2014 suggest the usefulness of measuring blood pressure at home for improving treatment continuation rate, evaluating the effectiveness of antihypertensive medications on decreasing blood pressure, and determining an effective treatment plan [1]. Therefore, it is critical for patients with hypertension to perform self-management behaviors, such as regular measurement of blood pressure at home. Consequently, nurses are required to comprehend patients' level of understanding of their disease, insufficient parts of self-management, and patients' feelings. Based on this information, nurses can then create a cooperative system by working with physicians to identify the contents and methods of patient education to enable them to continue appropriate self-management according to their individual abilities. In addition, nurses need to encourage patients to bring their family members to medical examinations and implement educational sessions that include family members so that they also understand the disease, self-management, and the importance of support from the family. Finally, to enhance patients' self-management, it is important to make patients and their families feel that nurses will always be available to support them.

\section{Conclusion}

A survey was conducted with patients with hypertension treated at an outpatient clinic to examine their understanding of the disease and self-management behavior. When considering the association between understanding of the disease, self-management behavior, and basic attributes, participants who reported that they understood the disease and the need to achieve their target blood pressure tended to exhibit more self-management behaviors, including measuring blood pressure at home, visiting physicians regularly, and consulting with physicians and nurses. In addition, participants who had a spouse and lived with their family had a higher rate of understanding the need to achieve their target blood pressure. Measuring and recording blood pressure are the most important self-management behaviors. To maintain appropriate self-management, it is essential that patients have an optimal understanding of the disease and self-management behaviors, and they receive support from their family. Therefore, the present findings emphasize the importance of the role of nurses, in cooperation with physicians, in providing patient education to improve their understanding of the disease and self-management, and including their families in discussions as much as possible.

\section{Acknowledgements}

The author is grateful to the patients who cooperated in this research and the doctors of the Department of Cardiologyat Tokyo Medical University Hospital.

\section{Conflicts of Interest}

The author declares no conflicts of interest regarding the publication of this paper. 


\section{References}

[1] The Japan Society of Hypertension Association Hypertension Treatment Guidelines Creation Committee (2014) Japan Society of Hypertension Treatment Guidelines, Japan Hypertension Society, Tokyo. https://www.jpnsh.jp/data/jsh2014/jsh2014v1 1.pdf

[2] Ohkubo, T., Asayama, K., Kikuya, M., Metoki, H., Hoshi, H., Hashimoto, J., Totsune, K., Satoh, H. and Imai, Y. (2004) How Many Times Should Blood Pressure Be Measured at Home for Better Prediction of Stroke Risk Ten-Year Follow-Up Results from the Ohasama Study. Journal of Hypertension, 22, 1099-1104. https://doi.org/10.1097/00004872-200406000-00009

[3] Sega, R., Facchetti, R., Bombelli, M., Cesana, G., Corrao, G., Grassi, G. and Mancia, G. (2005) Prognostic Value of Ambulatory and Home Blood Pressures Compared with Office Blood Pressure in the General Population: Follow-Up Results from the Pressioni Arteriose Monitorate Loro Associazioni (PAMELA) Study. Circulation, 111, 1777-1783. https://doi.org/10.1161/01.CIR.0000160923.04524.5B

[4] Stergiou, G.S., Nasothimiou, E.G., Kalogeropoulos, P.G., Pantazis, N. and Baibas, N.M. (2010) The Optimal Home Blood Pressure Monitoring Schedule Based on the Didima Outcome Study. Journal of Human Hypertension, 24, 158-164.

https://doi.org/10.1038/jhh.2009.54

[5] Niiranen, T.J., Johansson, J.K. and Reunanen, A. (2011) Optimal Schedule for Home Blood Pressure Measurement Based on Prognostic Data: The Finn-Home Study. Hypertension, 57, 1081-1086.

https://doi.org/10.1161/HYPERTENSIONAHA.110.162123

[6] Hozawa, A., Ohkubo, T., Nagai, K., et al. (2000) Prognosis of Isolated Systolic and Isolated Diastolic Hypertension as Assessed by Self-Measurement of Blood Pressure at Home the Ohasama Study. Archives of Internal Medicine, 160, 3301-3306. https://doi.org/10.1001/archinte.160.21.3301

[7] Asayama, K., Ohkubo, T., Kikuya, M., Metoki, H., Hoshi, H., Hashimoto, J., Totsune, K., Satoh, H. and Imai, Y. (2004) Prediction of Stroke by Self-Measurement of Blood Pressure at Home versus Casual Screening Blood Pressure Measurement in Relation to the Joint National Committee 7 Classification: The Ohasama Study. Stroke, 35, 2356-2361. https://doi.org/10.1161/01.STR.0000141679.42349.9f

[8] Nishinaga, M., Takata, J., Okumiya, K., Matsubayashi, K., Ozawa, T. and Doi, Y. (2005) High Morning Home Blood Pressure Is Associated with a Loss of Functional Independence in the Community-Dwelling Elderly Aged 75 Years or Older. Hypertension Research, 28, 657-663. https://doi.org/10.1291/hypres.28.657

[9] Agarwal, R. and Andersen, M.J. (2006) Prognostic Importance of Clinic and Home Blood Pressure Recordings in Patients with Chronic Kidney Disease. Kidney International, 69,406-411. https://doi.org/10.1038/sj.ki.5000081

[10] Imai, Y., Ohkubo, T., Hozawa, A., et al. (2001) Usefulness of Home Blood Pressure Measurements in Assessing the Effect of Treatment in a Single-Blind Placebo-Controlled Open Trial. Journal of Hypertension, 19, 179-185. https://doi.org/10.1097/00004872-200102000-00003

[11] Okubo, T., Imai, Y. and Nagai, K. (1998) Home Blood Pressure Measurement Has a Stronger Predictive Power for Mortality than Does Screening Blood Pressure Measurement: A Population-Based Observation in Ohasama, Japan. Journal of Hypertension, 16, 971-975. https://doi.org/10.1097/00004872-199816070-00010

[12] Kubota, Y., Heiss, G., Richard, F., et al. (2017) Association of Educational Attainment with Lifetime Risk of Cardiovascular Disease: The Atherosclerosis Risk in 
Communities Study. JAMA Internal Medicine, 177, 1165-1172. https://doi.org/10.1001/jamainternmed.2017.1877

[13] Makaya, M. (2014) Super-Elderly Heart Failure Care. Internal Medicine, 113, 505-509.

[14] Takada, A. (2012) The Processes and Characteristics between the Practice and Maintenance of Self-Management for Chronic Diseases and Response Shift Phenomenon. The Japanese Journal of Health Behavioral Science, 27, 140-156.

[15] Sasaki, C. (2013) The Relationship between General Self-Efficacy and Self Management and Health Related QOL in Patients with Chronic Heart Failure, University of Kindai Himeji. Bulletin of the Faculty of Nursing, 5, 21-30.

[16] Kitamura, Y. (2014) Actual Condition Correlated with Experience, Self Management, Self Efficacy and Quality of Life: Findings from Postoperative Gastroenterological Cancer Patients Receiving Outpatient Chemotherapy. Journal of Japanese Society of Cancer Nursing, 28, 13-23.

[17] Yamada, M. (2014) Situation of Self-Management of Chronic Obstructive Pulmonary Disease (COPD) Patients at Home: Analysis of the Physical State after One Year and Self-Management of One Year. The Annual Bulletin of Niigata College of Nursing, 3, 1-7.

[18] Hara, Y., Mastuoka, M., et al. (2006) Psychosocial Factors Associated with Treatment Satisfaction and Self-Esteem in Adults with Diabetes an Analysis on Family Support. Journal of Japan Academy of Diabetes Education and Nursing, 10, 4-15.

[19] Ministry of Health, Labour and Welfare (2016) Fiscal Year National Health Care Costs Overview. https://www.mhlw.go.jp/toukei/list/37-21.html 